\title{
Identification and characterization of microRNA sequences from bovine mammary epithelial cells
}

\author{
D. P. Bu, ${ }^{*} \dagger \ddagger^{1}$ X. M. Nan, ${ }^{*} \ddagger^{1}$ F. Wang, ${ }^{*} \dagger$ J. J. Loor,$\S^{2}$ and J. Q. Wang ${ }^{* 2}$ \\ *State Key Laboratory of Animal Nutrition, Institute of Animal Science, Chinese Academy of Agricultural Science, Beijing, 100193, China \\ †Chinese Academy of Agricultural Sciences (CAAS)-International Centre for Research in Agroforestry (ICRAF), World Agroforestry Centre, \\ East and Central Asia, Beijing 100081, China \\ $\ddagger$ Synergetic Innovation Center of Food Safety and Nutrition, Harbin, 150030, China \\ $\S$ Department of Animal Sciences and Division of Nutritional Sciences, University of Illinois, Urbana 61801
}

\section{ABSTRACT}

The bovine mammary gland is composed of various cell types including bovine mammary epithelial cells (BMEC). The use of BMEC to uncover the microRNA (miRNA) profile would allow us to obtain a more specific profile of miRNA sequences that could be associated with lactation and avoid interference from other cell types. The objective of this study was to characterize the miRNA sequences expressed in isolated BMEC. The miRNA were identified by Solexa sequencing technology (Illumina Inc., San Diego, CA). Furthermore, novel miRNA were uncovered by stem-loop reverse transcription-PCR and sequencing of PCR products. To detect tissue specificity, expression of novel miRNA sequences was measured by stem-loop RT-PCR and sequencing of PCR products in mammary gland, liver, adipose, ileum, spleen and kidney tissue from 3 lactating Holstein cows $(50 \pm 10 \mathrm{~d}$ postpartum). After bioinformatics analysis, 12,323,451 reads were obtained by Solexa sequencing, of which 11,979,706 were clean reads, matching the bovine genome. Among clean reads, $9,428,122$ belonged to miRNA sequences. Further analysis revealed that the miRNA bta-mir-184 had the most abundant expression, and 388 loci possessed the typical stem-loop structures matching known miRNA hairpins. In total, 38 loci with novel hairpins were identified as novel miRNA and were numbered from bta-U1 to bta-U38. One novel miRNA (bta-U21) was specific to mammary gland. Seven novel miRNA, including btaU21, had tissue-restricted distribution. Uncovering the specific roles of these novel miRNA during lactation appears warranted.

Key words: bovine mammary epithelial cell, novel microRNA, sequencing

\footnotetext{
Received April 8, 2014.

Accepted November 22, 2014.

${ }^{1}$ These authors contributed equally to this work.

${ }^{2}$ Corresponding authors: jloor@illinois.edu and jqwangcaas@gmail. com
}

\section{INTRODUCTION}

MicroRNA sequences (miRNA) are a class of small RNA molecules ( $\sim 22$ nucleotides, $\mathbf{n t})$ that inhibit translation or induce degradation of protein-coding mRNA containing sequences complementary to mRNA (Ambros, 2001; Bartel, 2004; Valencia-Sanchez et al., 2006). In nonruminants, these molecules play important roles in many specific biological processes such as apoptosis (Lewis et al., 2003; Liang et al., 2012), cell cleavage, proliferation and cell differentiation (Trompeter et al., 2011; Li and He, 2012), development (Bae et al., 2012), protein secretion (Mello and Czech, 2004), virus-host interaction (Wang et al., 2011; Skalsky et al., 2012), tumorigenesis or tumor inhibition (EsquelaKerscher and Slack, 2006; He et al., 2007; Jiang et al., 2012), DNA methylation or histone modification (Bao et al., 2004), and metabolism (Mersey et al., 2005; Zhu et al., 2011). It is estimated that 1 to $5 \%$ of all mammalian genes encode miRNA, thus potentially regulating the expression of approximately $30 \%$ of all protein-coding genes (Lewis et al., 2003; Lim et al., 2003). Each miRNA regulates, on average, 200 target genes through interactions between the seed sequence and the complementary target sites (Krek et al., 2005). According to miRBase 20 (http://www.mirbase.org/), the number of registered miRNA sequences has grown rapidly in recent years and the current total is 30,424 . However, compared with research in human, mouse, or other model organisms, little is known about miRNA in ruminants.

Although the identification and characterization of some miRNA in mammary (Li et al., 2012b; Naeem et al., 2012; Wang et al., 2012), adipose tissue (Jin et al., 2009, 2010; Romao et al., 2012), longissimus dorsi muscle (Jin et al., 2009; Sun et al., 2013), kidney (Jin et al., 2009), liver (Jin et al., 2009), spleen (Jin et al., 2009), thymus (Jin et al., 2009), cerebellum (Jin et al., 2009), medulla (Jin et al., 2009), hypothalamus (Jin et al., 2009), testicle (Huang et al., 2011), ovary (Hossain et al., 2009; Huang et al., 2011), embryo (Castro et al., 2010), and milk (Hata et al., 2010; Izumi et al., 2012) 
of bovine have been reported, to our knowledge, only a few studies describe miRNA profiles specific to bovine mammary epithelial cells (BMEC; Jin et al., 2014; Le Guillou et al., 2014).

Mammary cells or explants have been widely used over the years as models to understand the physiological function of the mammary gland. For instance, we established a functional BMEC line from Chinese Holstein cattle that exhibits normal extracellular matrix and is physiologically responsive to hormones ( $\mathrm{Hu}$ et al., 2009). This cell culture model was applied in an investigation of lactation in Chinese Holstein and in comparative studies of mammary function across cattle breeds (Cui et al., 2012; Nan et al., 2014). Therefore, BMEC represent a suitable model to characterize miRNA that are uniquely expressed in these cells. The specific objective of the present study was to use deepsequencing technology and bioinformatics to profile small RNA sequences (sRNA) in BMEC.

\section{MATERIALS AND METHODS}

All animal use was carried out in strict accordance with the Directions for Caring of Experimental Animals from the Ministry of Science and Technology, China. All efforts were made to minimize suffering.

\section{Cell Culture}

Isolation and characterization of BMEC has been described previously. Briefly, karyotype of the chromosome, protein expression of cytokeratin and $\beta$-casein, and mRNA expression of $A C A C A, B T N 1 A 1$, and $C S N 2$ were detected to characterize the cells (Hu et al., 2009). Cells preserved in liquid nitrogen with $10 \%$ dimethyl sulfoxide and $90 \%$ fetal calf serum were warmed in a $37^{\circ} \mathrm{C}$ water bath to recover cell viability. Subsequently, the BMEC were plated at $3 \times 10^{4}$ cells $/ \mathrm{cm}^{2}$ and incubated in 35-mm plastic dishes for $72 \mathrm{~h}$ until cell attachment could be verified. The confluent BMEC were incubated with Dulbecco's modified Eagle medium/nutrient mixture F-12 (DMEM/F12, GIBCO/Life Technologies, Grand Island, NY) supplemented with $0.5 \mu \mathrm{g} /$ $\mathrm{mL}$ insulin (Sigma Chemical Co., St. Louis, MO), 10 $\mathrm{ng} / \mathrm{mL}$ epidermal growth factor (Sigma Chemical Co.), $5 \mu \mathrm{g} / \mathrm{mL}$ transferrin (Sigma Chemical Co.), $1 \mu \mathrm{g} / \mathrm{mL}$ hydrocortisone (Sigma Chemical Co.), $1 \mu \mathrm{g} / \mathrm{mL}$ progesterone (Sigma Chemical Co.), $5 \mu \mathrm{g} / \mathrm{mL}$ estradiol, and $5 \mu \mathrm{g} / \mathrm{mL}$ prolactin for $48 \mathrm{~h}$ at $38^{\circ} \mathrm{C}$ and $5 \% \mathrm{CO}_{2}$. Subsequently, cells were washed with D-Hanks' buffer without $\mathrm{Ca}^{2+}$ or $\mathrm{Mg}^{2+}$ (contained $137 \mathrm{mM} \mathrm{NaCl}, 5.37$ $\mathrm{m} M \mathrm{KCl}, 0.34 \mathrm{~m} M \mathrm{Na}_{2} \mathrm{HPO}_{4} \cdot \mathrm{H}_{2} \mathrm{O}, 0.44 \mathrm{~m} M \mathrm{KH}_{2} \mathrm{PO}_{4}$, and $4.17 \mathrm{mM} \mathrm{NaHCO} 3$, with $\mathrm{pH}$ adjusted to 7.2-7.4) 3 times, and TRIzol reagent (Invitrogen, Carlsbad, CA) was added before miRNA isolation.

\section{Library Construction and Sequencing}

Total RNA from BMEC was isolated using TRIzol reagent, and the quality of RNA samples was assessed with an 2100 Bioanalyzer (Agilent, Santa Clara, CA) using the RNA6000 Nano LabChip Kit (Agilent). The RNA integrity number of the samples used was $\geq 8.0$. The RNA samples were then subjected to $15 \%$ PAGE and the RNA with length of 18 to 30 nt were isolated and purified (GeBAflex-tube Kit, GeBA, Geba, Israel). The $3^{\prime}$ and $5^{\prime}$ adapters (Illumina Inc., San Diego, CA) were ligated to the sRNA with T4 RNA ligase (New England Biolabs Inc., Ipswich, MA), and ligated products were subjected to reverse transcription (SuperScript II Reverse Transcriptase, Invitrogen). Complementary DNA was purified with $6 \%$ Novex Tris-borate-EDTA PAGE (Invitrogen) to construct a library. The quality of the library was validated by using the Qubit dsDNA HS Assay Kit (Invitrogen) in a Qubit 2.0 Fluorometer (Invitrogen), and by using the High Sensitivity DNA Reagent Kit (Agilent) in an Agilent 2100 Bioanalyzer. The resulting sRNA libraries were sequenced by Illumina/Solexa $1 \times 36$ bp sequencing at Beijing Genomics Institute (Shenzhen, China). Original reads and cluster density were justified to meet the quality of control standard (which was no less than $24 \mathrm{M}$ and $370 \mathrm{k}$, respectively).

\section{Analysis of Sequencing Data}

The 50-nt sequence tags from sequencing underwent data cleaning, which included removing reads of low quality, reads with $5^{\prime}$ primer contaminants, reads without $3^{\prime}$ primer, reads without the insert tag, reads with poly A, and reads shorter than $18 \mathrm{nt}$. Subsequently, the length distribution of the clean reads was summarized. Standard bioinformatics analyses were then used to annotate the clean tags into different categories.

The clean sRNA tags were mapped to the bovine genome (Btau 4.0) by SOAP (short oligonucleotide analysis package, http://soap.genomics.org.cn/; Li et al., $2008,2010)$ to analyze their expression and distribution in the genome. Small RNA tags with rRNA, small cytosol RNA (scRNA), small nucleolar RNA (snoRNA), small nuclear RNA (snRNA), Piwi-interacting RNA (piRNA), and transfer RNA (tRNA) were annotated with GenBank, and matched tags were separated from unannotated tags. The sRNA were also compared with the miRNA precursor of Bos taurus (or mature miRNA if there was no precursor information) to identify known miRNA sequences. The reading count and base bias on 
Table 1. Analysis of fragment types

\begin{tabular}{lrc}
\hline $\begin{array}{l}\text { Type of } \\
\text { fragments }\end{array}$ & $\begin{array}{c}\text { Abundance } \\
(\text { no. })\end{array}$ & $\begin{array}{c}\text { Frequency } \\
(\%)\end{array}$ \\
\hline Total reads & $12,323,451$ & \\
High quality & $12,297,272$ & 100 \\
3' adapter null & 18,793 & 0.15 \\
Insert null & 263,811 & 2.12 \\
5' adapter contaminants & 6,881 & 0.06 \\
Smaller than 18 nt & 28,026 & 0.23 \\
Poly A & 55 & 0.00 \\
Clean reads & $11,979,706$ & 97.42 \\
\hline
\end{tabular}

the first position of identified miRNA of certain length were analyzed. The sRNA tags were then aligned with exons and introns of mRNA to find degraded fragments of mRNA in the sRNA tags.

In the alignment and annotation process described above, some sRNA tags were mapped to more than one category. To ensure that every sRNA was mapped to only one annotation term, we used the following priority rule: rRNA > known miRNA > repeat $>$ exon $>$ intron (Calabrese et al., 2007). The total rRNA proportion is a mark of sample quality. For high-quality samples, it should be less than $60 \%$ in plant samples and $40 \%$ in animal samples.

The characteristic hairpin structure of miRNA precursor can be used to predict novel miRNA (Huang et al., 2011). We used MIREAP (http://sourceforge. net/projects/mireap/), which is a suitable software tool to predict novel miRNA in vertebrates ( $\mathrm{Li}$ et al., 2012a), by exploring the secondary structure, the Dicer cleavage site, and the minimum free energy (MFE) of the unannotated sRNA tags, which could be mapped to the genome. The rules used for prediction were as reported by Huang et al. (2011) and considered factors including minimal and maximal miRNA sequence length, minimal depth of Drosha/Dicer cutting site, maximal copy number of miRNA on reference, MFE allowed for a miRNA precursor, maximal space between miRNA and miRNA*, minimal base pairs and maximal bulge of miRNA and miRNA*, maximal asymmetry of miRNA/miRNA* duplex (where miRNA* represents the sequence with the lower expression level), and the sequence length of flank region in miRNA precursor. The base bias of predicted novel miRNA candidates was summarized and the expression of novel miRNA sequences in BMEC was further validated by quantitative real-time (qRT)-PCR and sequencing of the PCR products.

\section{Tissue Sample Preparation}

Three lactating Chinese Holstein cows $(50 \pm 10 \mathrm{~d}$ postpartum) in good health from the dairy farm of the Chinese Academy of Agricultural Sciences (Beijing) were euthanized according to the rules from Directions for Caring of Experimental Animals from the Ministry of Science and Technology, China. After death, mammary tissue, liver, adipose, spleen, ileum, and rumen papillae samples were collected. Tissue samples used for the analysis of miRNA were washed with PBS prepared with RNase-free water, hydrated, and then immediately stored in liquid nitrogen until RNA extraction.

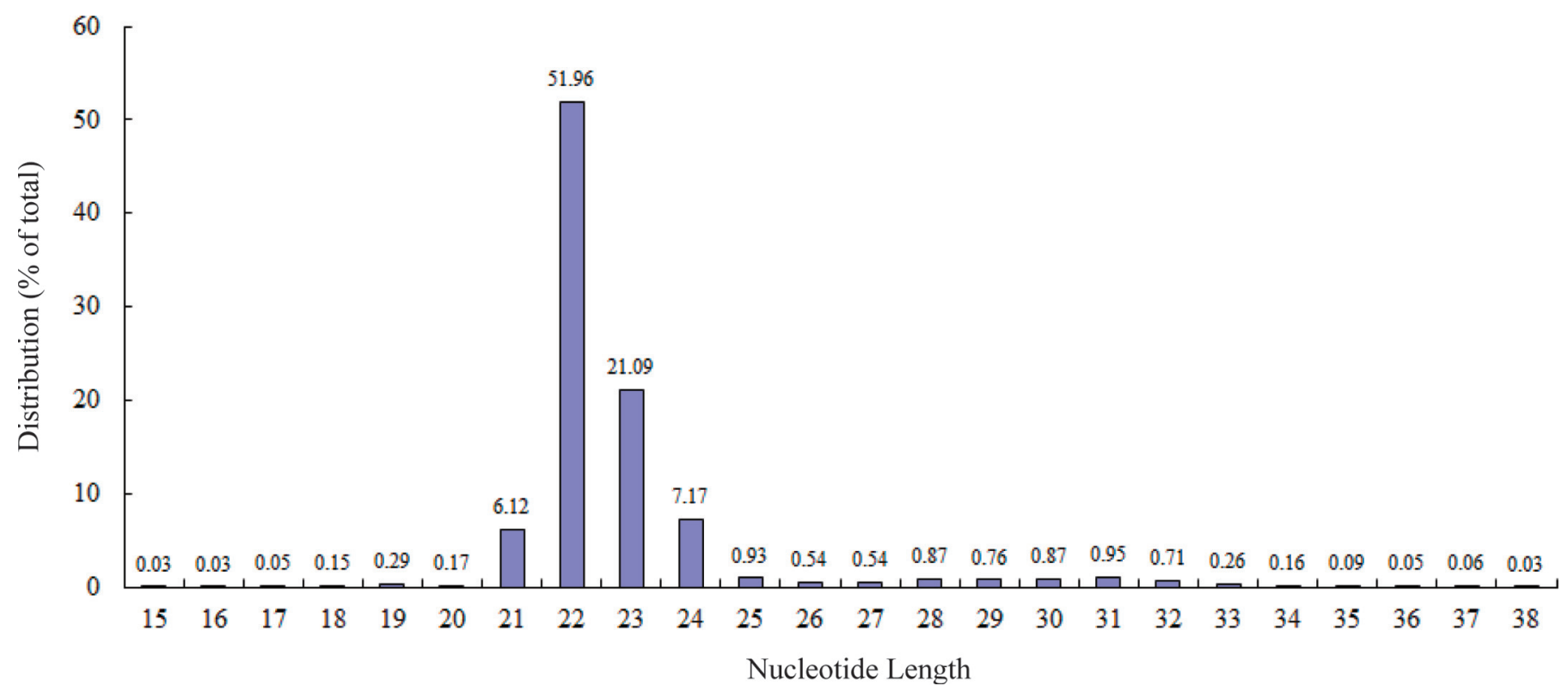

Figure 1. Distribution (\%) of small RNAs detected. Color version available online. 
(a)

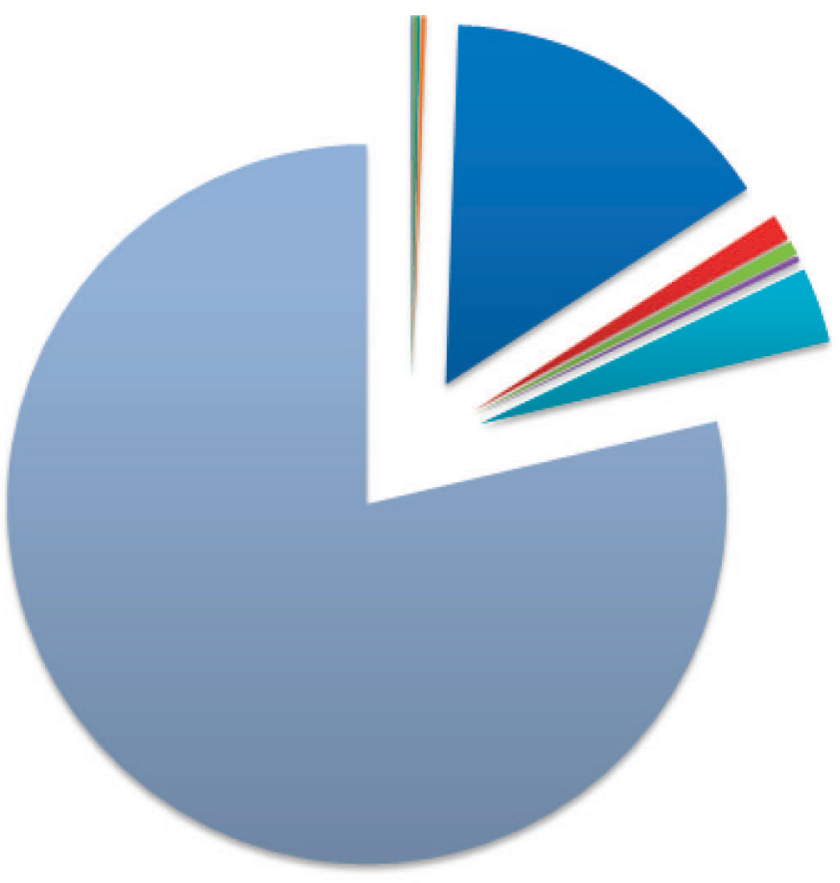

(b)

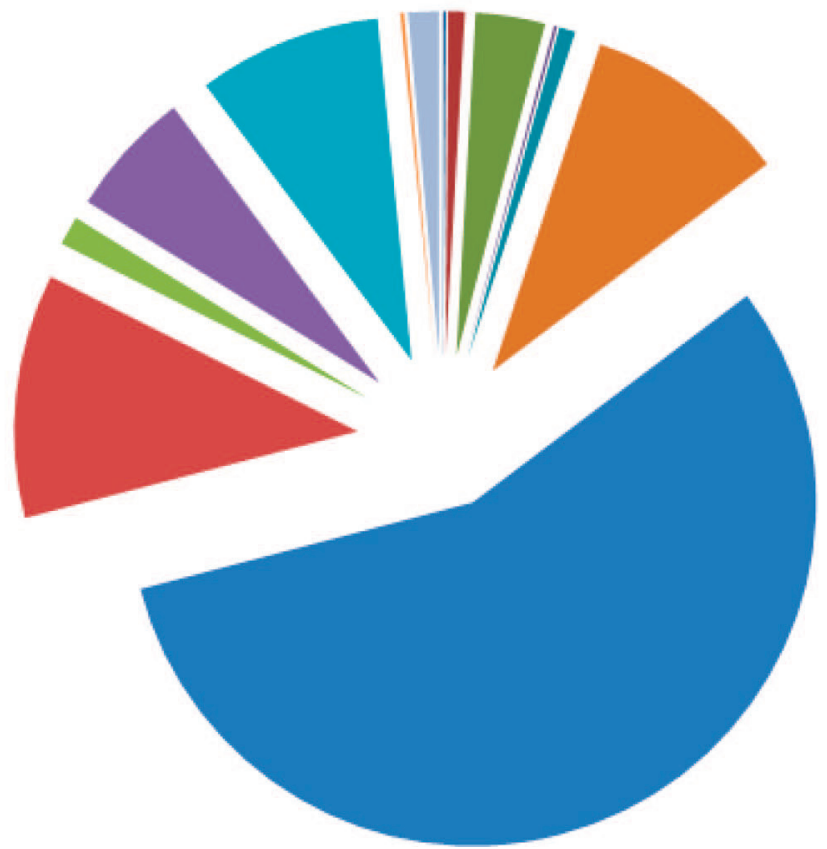

m scRNA (0.060\%)

Eintron-antisense $(0.019 \%)$

Eintron-sense (0.168\%)

m $\operatorname{srpRNA}(0.005 \%)$

m $\operatorname{snRNA}(0.116 \%)$

Wex on_sense (0.191\%)

=unann $(15.270 \%)$

ERNA (1.204\%)

m snoRNA $(0.62 \%)$

vepeat $(0.268 \%)$

atRA $(3.376 \%)$

W ex on_antisense $(0.004 \%)$

$=\operatorname{miRNA}(78.701 \%)$

I $\operatorname{scRNA}(0.125 \%)$

intron_antisense $(0.770 \%)$

mintron_sense (3.270\%)

merpA $(0.140 \%)$

= snRNA (0.764\%)

Ex on_sense ( $9.656 \%)$

- unann $(56.217 \%)$

= rRNA (11.503\%)

snoRNA (1.376\%)

- repeat (5.950)

tRNA $(8.638 \%)$

W ex on_antisense $(0.159 \%)$

$=\operatorname{miRNA}(1.432 \%)$

Figure 2. Total (a) and bovine-specific (b) annotation categories. scRNA = small cytosol RNA; srpRNA = signal recognition particle RNA; snRNA $=$ small nuclear RNA; tRNA = transfer RNA; snoRNA = small nucleolar RNA; miRNA $=$ microRNA; unann $=$ unannotated .

\section{RNA Extraction, Reverse Transcription, and PCR of Tissue Samples}

Total RNA from mammary, liver, adipose, spleen, ileum, and rumen papillae samples was isolated using TRIzol reagent. The quality and quantity of RNA samples were examined using an Agilent 2100 Bioana- lyzer with the RNA6000 Nano Lab Chip Kit (Agilent). Reverse transcription and PCR were performed with specific reverse transcription primers according to the miRNA sequence and SYBR Premix Ex TaqTM II (Perfect Real Time; TaKaRa, Tokyo, Japan). The reverse transcription primers and PCR primers used in this experiment are shown in Supplemental Table S1 


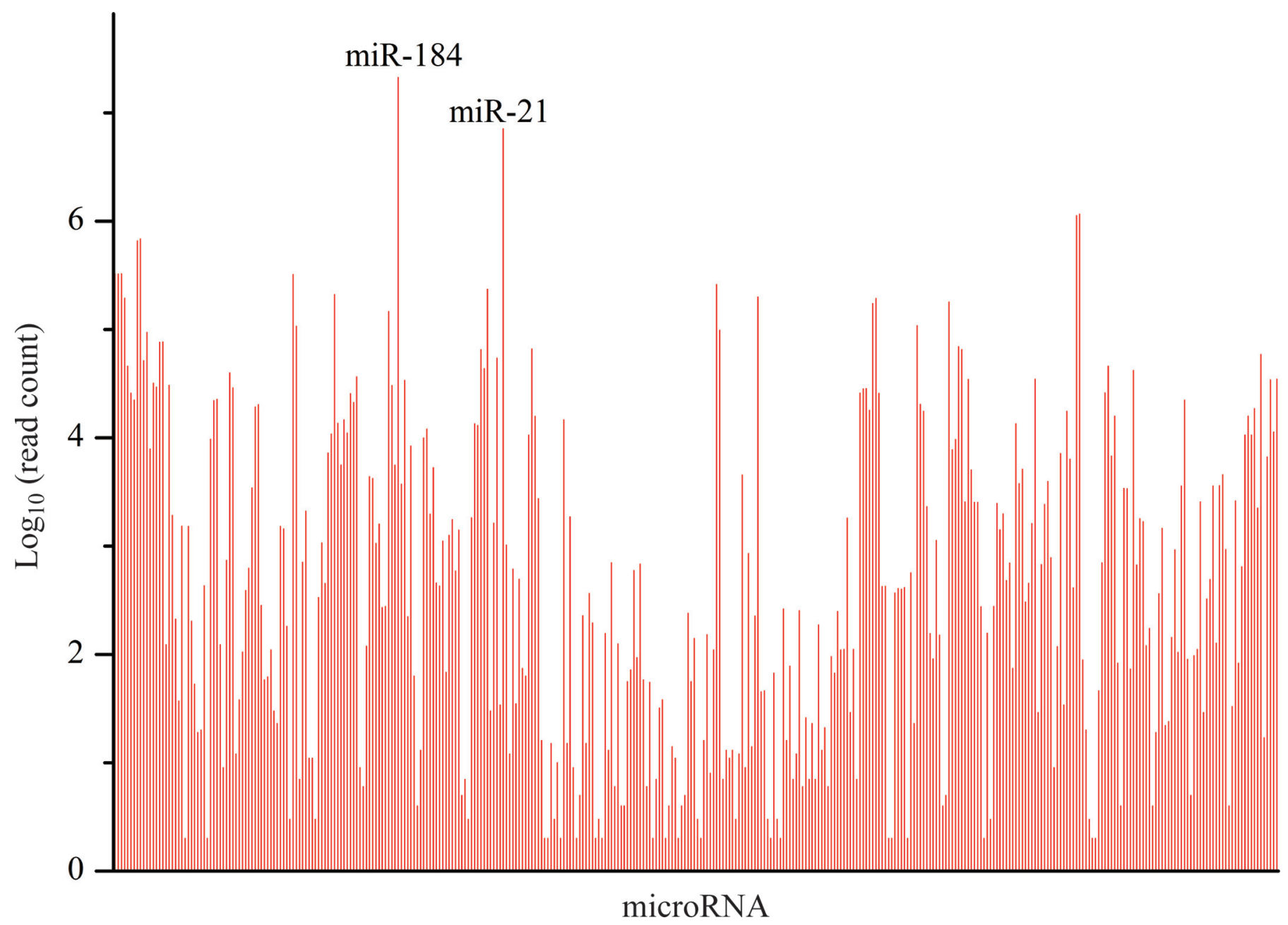

Figure 3. Read counts of 388 known microRNA sequences. Among all microRNA sequences detected, miR-184 and miR-21 had the highest expression. Color version available online.

(http://dx.doi.org/10.3168/jds.2014-8217). The PCR products were sequenced to define the validity.

\section{Amplicon Sequencing}

The PCR products with proper length were collected and ligated to the pUCm-T Vector (Sangon Biotech, China) with T4 DNA Ligase (Sangon Biotech, Shanghai, China). Ligation products were transfected to competent bacterial cells that were treated using a Single Step Competent Cell Prep Kit before the transfection (Sangon Biotech). Transformed products were then spread on ampicillin-containing culture plates that were coated with isopropyl $\beta$-D-1-thiogalactopyranoside and 5-bromo-4-chloro-3-indolyl $\beta$-D-galactopyranoside before use. The positive bacterial colonies were selected and plasmid DNA was extracted with the UNIQ-10 Column Plasmid Mini Prep Kit (Sangon Biotech).
After an amplification reaction, PCR products were sequenced by using the 3730xl DNA Analyzer (Applied Biosystems), and sequence alignment between amplicon and miRNA was performed with DNAssist (version 1.02, http://dnassist.en.softonic.com/).

\section{Target Prediction and Biological Process Analysis}

The target genes of one novel miRNA were predicted with miRnada 3.3a (http://www.microrna.org/microrna/getMirnaForm.do), and the target genes were annotated by using Gene Ontology annotations (http:// geneontology.org/). The cluster analysis of biological processes was performed using DAVID Bioinformatics Resources 6.7 (http://david.abcc.ncifcrf.gov/) and the methods referred to the work of Huang et al. (2009), which not only classify large gene lists into functionally related gene groups but also rank the importance of 
Table 2. Analysis of novel microRNA sequences

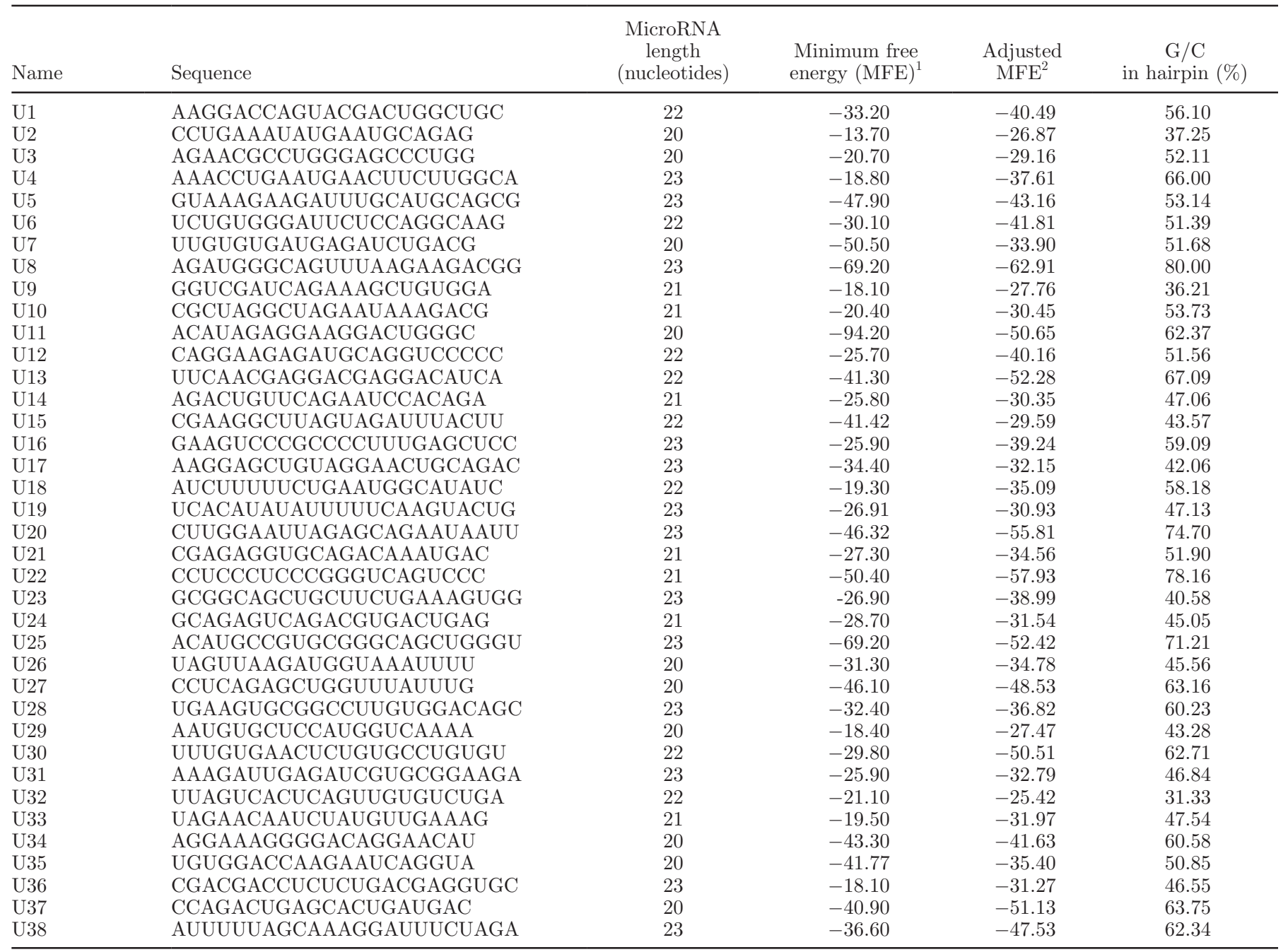

${ }^{1}$ The lower the value is, the more credible it is (i.e., the more likely it is to be a novel miRNA).

${ }^{2}$ Adjusted MFE $=$ MFE/length (hairpin) $\times 100$; thus, adjusted MFE shows MFE per $100 \mathrm{nt}$, allowing better comparison between sequences.

the discovered gene groups based on statistical $P$-values according to hypergeometric test and false discovery rate (FDR) correction method.

\section{RESULTS AND DISCUSSION}

Deep sequencing of the sRNA fraction within cells yields an incredibly rich amount of data from which we can determine not only the expression levels of miRNA but also the levels of other sRNA species such as piRNA or snoRNA, as well as discover novel sRNA sequences. The latter is one of the key advantages of sRNA sequencing (Creighton et al., 2009). Several resources supporting annotation, base bias, length bias, genome bias, and novel miRNA prediction have been reported (Oshlack and Wakefield, 2009; Frith et al.,
2010; Kozomara and Griffiths-Jones, 2011). Those tools were beneficial for the identification and characterization of miRNAs in BMEC.

Mammary gland is composed of various cell types such as epithelial (including mammary alveolar epithelium, ductal epithelium, and myoepithelium), connective, and adipose. Therefore, the use of BMEC to uncover the miRNA profile allowed us to obtain a more specific profile of miRNA that could be associated with lactation and avoid interference from other cell types. Solexa sequencing of BMEC resulted in 12,323,451 raw reads (Table 1), and, after removing the low-quality sequences, 12,297,272 high-quality reads were obtained. Furthermore, after excluding adapters and insufficiently tagged sequences, 11,979,706 clean reads were obtained (Table 1) and accounted for $97.04 \%$ of the high-quality 


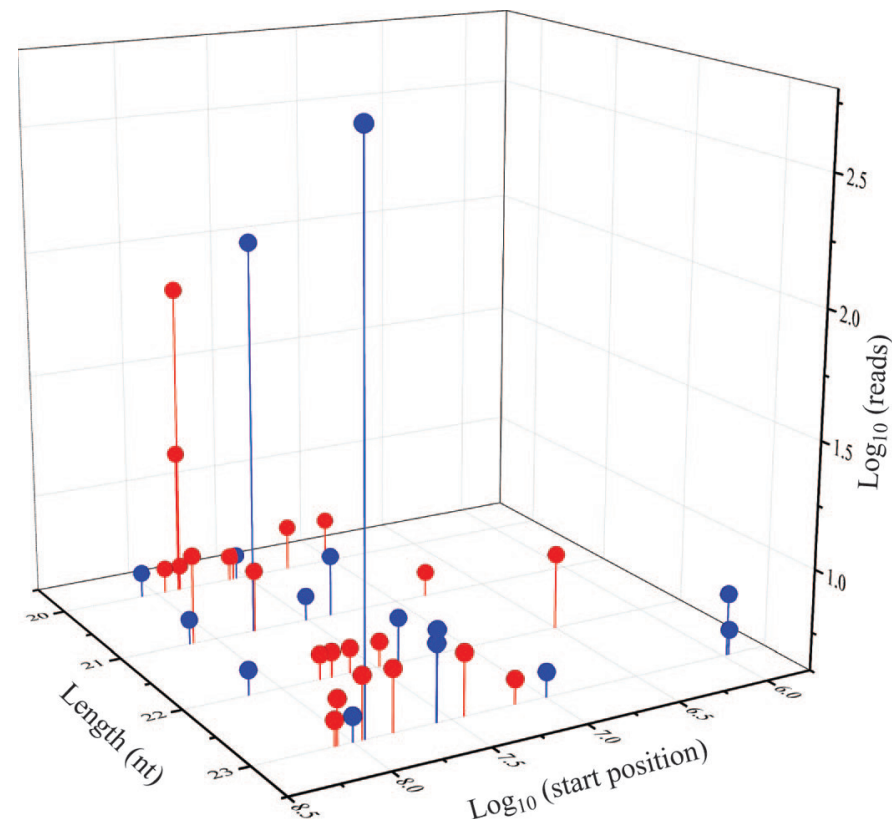

Figure 4. Length, read counts, and start position in chromosomes of 38 novel microRNA sequences. The read counts and the start position in the chromosomes were $\log _{10}$ transformed for ease of reporting. The (light gray or red) circles denote location in the plus chain, whereas (dark gray or blue) circles denote location in the minus chain. Color version available online.

reads, demonstrating that the sequencing data were of high quality and reliable.

The most abundant and diverse species of the sRNA (16-30 nt) in the library were those of length 21 to 24 nt, which is a typical size range for Dicer-derived products (Figure 1). In BMEC, the 22- and 23-nt sequences appeared to be the dominant type of sRNA, accounting for $51.96 \%$ and $21.09 \%$ of the total sequences, respectively (Figure 1).

In total, 10,014,804 reads were matched to the bovine genome (Btau_4.0) using the SOAP program. The total and unique bovine sRNA were annotated into different categories (Figure 2). Among these genome-matched reads, 9,428,122 reads belonged to miRNA expressed in BMEC (about $78 \%$ of the total reads) and conicided with the dominant proportion of 22 -nt sequences. In a recent study, the relative abundance of different classes of sRNA sequences in BMEC was detected and, as in the current study, about $76 \%$ of the total reads were miRNA (Jin et al., 2014). The percentage of rRNA sequences was much less than $40 \%$, which indicated the samples were of good quality. Unannotated sRNA within the bovine unique set were predominant, in accordance with the relatively less-studied bovine sRNA compared with that of other model organisms. Considering the miRNA sequences within the sRNA sequences in all gene-matched reads was advantageous, and the unannotated bovine-specific sRNA were very likely to be miRNA sequences.

To determine whether these sRNA sequences are genuine cattle miRNA, we scanned the bovine genome for hairpin structures comprising the candidate miRNA using MIREAP software, which can be used to identify both known and novel miRNA from sRNA libraries generated by the Solexa technology (Huang et al., 2011). In our sRNA library, 388 loci possessing the typical stem-loop structures were matched to known miRNA hairpins in the bovine genome. Among them, miR-184 had the most abundant expression (Figure 3) with $21,296,246$ reads, followed by miR-21. In another study on miRNA profiles in BMEC, miR-21 was most predominant (Jin et al., 2014), although miR-184 also had very high expression.

To date, the number of known miRNA detected in mammary gland or mammary epithelial cells differs among studies. For instance, Le Guillou et al. (2014) reported 167 known miRNA in bovine mammary gland whereas Jin et al. (2014) confirmed 231 in epithelial cells. MicroRNA family analysis can provide fundamental information about the degree of miRNA conservation and species specificity. According to our results, 13 of the families to which the known miRNA belong, including miR-2284, miR-2300, miR-2319, miR-2329, miR-2363, miR-2384, miR-2404, miR-2450, miR-2887, miR-2902, miR-2904, miR-3432 and miR-3604, are bovine specific and the others are most conserved among species. Details are reported in Supplemental Table S2 (spreadsheet; http://dx.doi.org/10.3168/jds.2014-8217).

The software MIREAP utilizes the typical stem-loop hairpin criterion to detect novel miRNA. A typical stem-loop hairpin should have the following characteristics: mature miRNA sequences located in one arm of the hairpin precursor without large internal loops or bulges; free energy of hybridization of the hairpins $<-18$ $\mathrm{kcal} / \mathrm{mol}$; and hairpins located in intergenic regions or introns (Huang et al., 2011). Using this approach, we identified 38 unannotated genes with novel sequences and structures, which were numbered sequentially from bta-U1 to bta-U38 (Table 2). The expression level and location in chromosomes are depicted in Figure 4. The results revealed that most of the novel miRNA had no length preference. Precursors of the novel miRNA sequences that were identified formed proper secondary hairpin structures.

Analyses of the first nucleotide bias of the 18- to 25-nt miRNA candidates revealed that uridine (U) was the most common nucleotide at the $5^{\prime}$ end of sRNA sequences of length $18,20,21$, and $22 \mathrm{nt}$, whereas adenine (A) was the most common first nucleotide of sRNA sequences of length 19, 23, 24, and 25 nt (Supplemental Figure S1; http://dx.doi.org/10.3168/jds.2014-8217). 
Table 3. Novel microRNA expression in 6 dairy cow tissues ${ }^{1}$

\begin{tabular}{|c|c|c|c|c|c|c|}
\hline MicroRNA & Mammary & Liver & Adipose & Spleen & Ileum & Rumen papillae \\
\hline Bta-U 1 & + & + & + & + & + & + \\
\hline Bta-U 2 & + & + & + & + & + & + \\
\hline Bta-U 3 & + & + & + & + & + & + \\
\hline Bta-U 4 & + & + & + & + & + & + \\
\hline Bta-U 5 & + & + & + & + & + & + \\
\hline Bta-U 6 & + & + & + & + & + & + \\
\hline Bta-U 7 & + & + & + & + & + & + \\
\hline Bta-U 8 & + & + & + & + & + & + \\
\hline Bta-U 9 & + & + & + & + & + & + \\
\hline Bta-U 10 & + & + & + & + & + & + \\
\hline Bta-U 11 & + & + & + & + & + & + \\
\hline Bta-U 12 & + & + & + & + & + & + \\
\hline Bta-U 13 & + & + & + & + & + & + \\
\hline Bta-U 14 & + & + & + & + & + & + \\
\hline Bta-U 15 & + & + & + & + & + & + \\
\hline Bta-U 16 & + & + & + & + & + & + \\
\hline Bta-U 17 & + & + & + & + & + & + \\
\hline Bta-U 18 & + & + & + & + & + & - \\
\hline Bta-U 19 & + & + & - & + & + & + \\
\hline Bta-U 20 & + & + & + & + & + & + \\
\hline Bta-U 21 & + & - & - & - & - & - \\
\hline Bta-U 22 & + & + & + & - & + & + \\
\hline Bta-U 23 & + & + & + & + & + & + \\
\hline Bta-U 24 & + & + & + & + & + & + \\
\hline Bta-U 25 & + & + & + & + & + & + \\
\hline Bta-U 26 & + & + & + & + & + & + \\
\hline Bta-U 27 & + & + & + & + & + & + \\
\hline Bta-U 28 & + & + & - & + & + & + \\
\hline Bta-U 29 & + & + & + & + & + & + \\
\hline Bta-U 30 & + & + & + & + & + & + \\
\hline Bta-U 31 & + & + & + & + & + & + \\
\hline Bta-U 32 & + & + & + & - & + & + \\
\hline Bta-U 33 & + & - & + & + & + & + \\
\hline Bta-U 34 & + & + & + & + & + & + \\
\hline Bta-U 35 & + & + & + & + & + & + \\
\hline Bta-U 36 & + & + & + & + & + & + \\
\hline Bta-U 37 & + & + & + & + & + & + \\
\hline Bta-U 38 & + & + & + & + & + & + \\
\hline
\end{tabular}

${ }^{1}$ Where + denotes detectable miRNA and - denotes undetectable miRNA.

Analyses of the first nucleotide bias of the 38 novel microRNA candidates revealed that $\mathrm{A}$ and $\mathrm{U}$ were the most common nucleotides at the $5^{\prime}$ end of the miRNA (Supplemental Figure S1), which is in accordance with the known miRNA. As such, it provides evidence for these miRNA being novel.

In accordance with sequencing results of BMEC, all 38 miRNA were detected in bovine mammary tissue (Table 3). In the tissue distribution analysis of the 38 novel miRNA, bta-U21 was found to be detectable only in mammary tissue, which proved the tissue specificity of this miRNA within the limited context of the study. Another 6 novel miRNA (including U18, U19, U22, U28, U32, and U33) were not detectable in some tissue samples (Table 3). Thus, the 6 novel miRNAs as well as bta-U21 had tissue-restricted distribution, suggesting that some of those may play unique roles in mammary function.
Target prediction is very important to understand the function of unique miRNA. Considering the tissue specificity of bta-U21 in mammary gland, its target genes were predicted and annotated, and the biological processes related to the genes were clustered and statistically scored based on DAVID Bioinformatics Resources 6.7. According to the enrichment $P$-value, 33 biological processes were uncovered $(P<0.05$, Figure $5)$, underscoring that bta-U21 has potential roles in molecular regulation, signal transduction, and metabolism. Further research is required to determine whether bta-U21 has specialized roles in mammary tissue.

In conclusion, the present study identified 388 known miRNA and 38 novel miRNA expressed in BMEC. Some had tissue specificity within the limited context of the study (e.g., bta-U21 in mammary gland) or tissuerestricted distribution, which not only provides the exact miRNA profile in lactating BMEC but also expands 


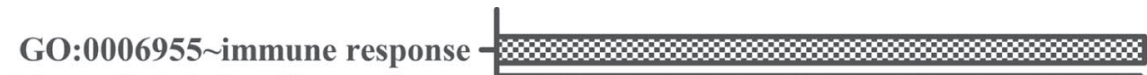

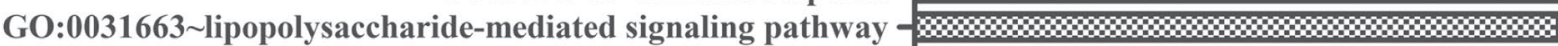

GO:0030030 cell projection organization $-8 \% 8 \% 8 \% 8 \% 8 \% 8 \% 8 \% 8 \% 8 \% 8 \% 8 \% 8 \% 8 \%$

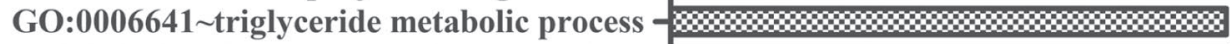

G0:0060191 regulation of lipase activity $-8 \times 8 \times 8 \times 8 \times 808 \times 8 \times 8 \times 8 \times 8 \times 8 \times 8 \times 8 \times 88$

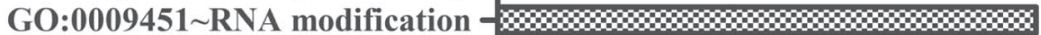

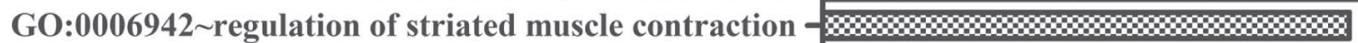

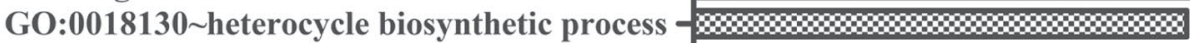

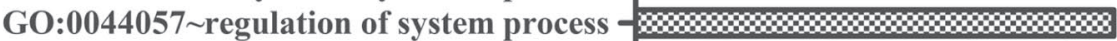

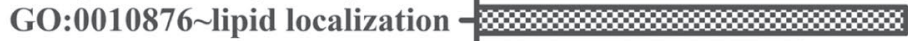

GO:0051336 regulation of hydrolase activity $-8 \% \% 8 \% \% \% \% 8 \%$

GO:0016125 sterol metabolic process $-\$ \times 8 \times 6 \% 8 \% 8 \% 8 \% 8 \%$

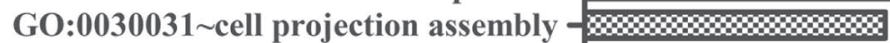

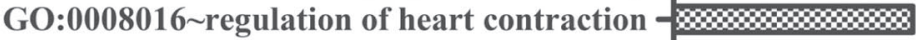

GO:0006869 lipid transport $-80808808 \% 88$

G0:0010885 regulation of cholesterol storage $-\$ \times 8 \% \% \% \% \%$

GO:0030035 microspike assembly $-\$ 0808 \% 8 \% 8$

GO:0016192 vesicle-mediated transport -8088088080

GO:0060193 positive regulation of lipase activity $-808088 \times 8 \times 8$

GO:0046847 filopodium assembly $\$ \times \infty \times 8 \times$

GO:0008015 blood circulation $-\$ \infty 8 \%$

GO:0003013 circulatory system process $-\$ \infty 8 \% 8 \%$

GO:0008104 protein localization $-8080 \times 80$

GO:0009792 embryonic development ending in birth or egg hatching $-\$ 28 \times 8 \times$

GO:0043009 chordate embryonic development $-\infty \times 8 \times 8$

GO:0008610 lipid biosynthetic process $-\infty 88$

GO:0051345 positive regulation of hydrolase activity $-\$ 8 \times$

GO:0007242 intracellular signaling cascade $-\infty$

GO:0045184 establishment of protein localization $-\infty$

GO:0015031 protein transport $-\infty$

GO:0007264 small GTPase mediated signal transduction $-\otimes$

GO:0043085 positive regulation of catalytic activity $-\varnothing$

GO:0044093 positive regulation of molecular function -

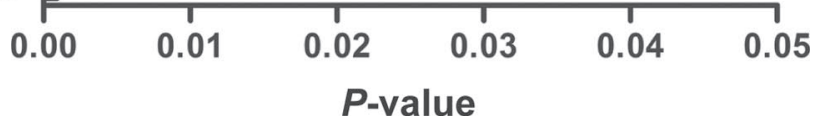

Figure 5. Biological process cluster analysis of predicted target genes of microRNA U21 (miR-U21). Predicted target genes of miR-U21 were classified into functionally related gene groups (biological processes), and the importance of the discovered gene groups was ranked based on statistical $P$-values. Biological processes with $P$-values $<0.05$ are reported in the figure. GO = gene ontology annotations (http://geneontology. $\operatorname{org} /)$.

our miRNA knowledge of Bos taurus. Uncovering their specific roles during lactation appears warranted.

\section{ACKNOWLEDGMENTS}

Financial support for this study was provided by National Key Basic Research Program of China (Beijing; No. 2011CB100805) and grants from Ministry of Science and Technology (Beijing, China; 2012BAD12B02-5) and the Agricultural Science and Technology Innovation Program (Beijing, China; ASTIP-IAS07).

\section{REFERENCES}

Ambros, V. 2001. microRNAs: Tiny regulators with great potential Cell 107:823-826.
Bae, Y., T. Yang, H. C. Zeng, P. M. Campeau, Y. Chen, T. Bertin, B C. Dawson, E. Munivez, J. Tao, and B. H. Lee. 2012. miRNA-34c regulates Notch signaling during bone development. Hum. Mol. Genet. 21:2991-3000.

Bao, N., K. W. Lye, and M. K. Barton. 2004. MicroRNA binding sites in Arabidopsis class III HD-ZIP mRNAs are required for methylation of the template chromosome. Dev. Cell 7:653-662.

Bartel, D. P. 2004. MicroRNAs: Genomics, biogenesis, mechanism, and function. Cell 116:281-297.

Calabrese, J. M., A. C. Seila, G. W. Yeo, and P. A. Sharp. 2007. RNA sequence analysis defines Dicer's role in mouse embryonic stem cells. Proc. Natl. Acad. Sci. USA 104:18097-18102.

Castro, F. O., S. Sharbati, L. L. Rodriguez-Alvarez, J. F. Cox, C. Hultschig, and R. Einspanier. 2010. MicroRNA expression profiling of elongated cloned and in vitro-fertilized bovine embryos. Theriogenology 73:71-85.

Creighton, C. J., J. G. Reid, and P. H. Gunaratne. 2009. Expression profiling of microRNAs by deep sequencing. Brief. Bioinform. 10:490-497. 
Cui, R. L., J. Q. Wang, H. Y. Wei, D. P. Bu, X. M. Nan, H. Hu, P. Sun, and L. Y. Zhou. 2012. Effects of 18-carbon fatty acids on triacylglycerol accumulation in bovine mammary epithelial cells in vitro. J. Dairy Sci. 95(Suppl. 2):347. (Abstr.)

Esquela-Kerscher, A., and F. J. Slack. 2006. Oncomirs-MicroRNAs with a role in cancer. Nat. Rev. Cancer 6:259-269.

Frith, M. C., R. Wan, and P. Horton. 2010. Incorporating sequence quality data into alignment improves DNA read mapping. Nucleic Acids Res. 38:e100.

Hata, T., K. Murakami, H. Nakatani, Y. Yamamoto, T. Matsuda, and N. Aoki. 2010. Isolation of bovine milk-derived microvesicles carrying mRNAs and microRNAs. Biochem. Biophys. Res. Commun. 396:528-533.

He, L., X. He, S. W. Lowe, and G. J. Hannon. 2007. microRNAs join the p53 network-Another piece in the tumour-suppression puzzle. Nat. Rev. Cancer 7:819-822.

Hossain, M. M., N. Ghanem, M. Hoelker, F. Rings, C. Phatsara, E. Tholen, K. Schellander, and D. Tesfaye. 2009. Identification and characterization of miRNAs expressed in the bovine ovary. BMC Genomics 10:443.

Hu, H., J. Wang, D. Bu, H. Wei, L. Zhou, F. Li, and J. J. Loor. 2009. In vitro culture and characterization of a mammary epithelial cell line from Chinese Holstein dairy cow. PLoS ONE 4:e7636.

Huang, D. W., B. T. Sherman, and R. A. Lempicki. 2009. Systematic and integrative analysis of large gene lists using DAVID bioinformatics resources. Nat. Protoc. 4:44-57.

Huang, J., Z. Ju, Q. Li, Q. Hou, C. Wang, J. Li, R. Li, L. Wang, T. Sun, S. Hang, Y. Gao, M. Hou, and J. Zhong. 2011. Solexa sequencing of novel and differentially expressed microRNAs in testicular and ovarian tissues in Holstein cattle. Int. J. Biol. Sci. 7:1016-1026.

Izumi, H., N. Kosaka, T. Shimizu, K. Sekine, T. Ochiya, and M. Takase. 2012. Bovine milk contains microRNA and messenger RNA that are stable under degradative conditions. J. Dairy Sci. 95:4831-4841.

Jiang, W., X. Chen, M. Liao, W. Li, B. Lian, L. Wang, F. Meng, X. Liu, Y. Jin, and X. Li. 2012. Identification of links between small molecules and miRNAs in human cancers based on transcriptional responses. Sci. Rep. 2:282.

Jin, W., M. V. Dodson, S. S. Moore, J. A. Basarab, and L. L. Guan. 2010. Characterization of microRNA expression in bovine adipose tissues: A potential regulatory mechanism of subcutaneous adipose tissue development. BMC Mol. Biol. 11:29.

Jin, W., J. R. Grant, P. Stothard, S. S. Moore, and L. L. Guan. 2009. Characterization of bovine miRNAs by sequencing and bioinformatics analysis. BMC Mol. Biol. 10:90.

Jin, W., E. M. Ibeagha-Awemu, G. Liang, F. Beaudoin, X. Zhao, and L. L. Guan. 2014. Transcriptome microRNA profiling of bovine mammary epithelial cells challenged with Escherichia coli or Staphylococcus aureus bacteria reveals pathogen directed microRNA expression profiles. BMC Genomics 15:181.

Kozomara, A., and S. Griffiths-Jones. 2011. miRBase: Integrating microRNA annotation and deep-sequencing data. Nucleic Acids Res. 39:D152-D157.

Krek, A., D. Grun, M. N. Poy, R. Wolf, L. Rosenberg, E. J. Epstein, P. MacMenamin, I. da Piedade, K. C. Gunsalus, M. Stoffel, and N. Rajewsky. 2005. Combinatorial microRNA target predictions. Nat. Genet. 37:495-500.

Le Guillou, S., S. Marthey, D. Laloe, J. Laubier, L. Mobuchon, C. Leroux, and F. Le Provost. 2014. Characterisation and comparison of lactating mouse and bovine mammary gland miRNomes. PLoS ONE 9:e91938.

Lewis, B. P., I. H. Shih, M. W. Jones-Rhoades, D. P. Bartel, and C. B. Burge. 2003. Prediction of mammalian microRNA targets. Cell 115:787-798.

Li, M. A., and L. He. 2012. microRNAs as novel regulators of stem cell pluripotency and somatic cell reprogramming. BioEssays 34:670-680.

Li, R., Y. Li, K. Kristiansen, and J. Wang. 2008. SOAP: Short oligonucleotide alignment program. Bioinformatics 24:713-714.
Li, R., H. Zhu, J. Ruan, W. Qian, X. Fang, Z. Shi, Y. Li, S. Li, G. Shan, K. Kristiansen, H. Yang, and J. Wang. 2010. De novo assembly of human genomes with massively parallel short read sequencing. Genome Res. 20:265-272.

Li, Y., Z. Zhang, F. Liu, W. Vongsangnak, Q. Jing, and B. Shen. 2012a. Performance comparison and evaluation of software tools for microRNA deep-sequencing data analysis. Nucleic Acids Res. 40:4298-4305.

Li, Z., H. Liu, X. Jin, L. Lo, and J. Liu. 2012b. Expression profiles of microRNAs from lactating and non-lactating bovine mammary glands and identification of miRNA related to lactation. BMC Genomics 13:731.

Liang, X., D. Zhou, C. Wei, H. Luo, J. Liu, R. Fu, and S. Cui. 2012. MicroRNA-34c enhances murine male germ cell apoptosis through targeting ATF1. PLoS ONE 7:e33861.

Lim, L. P., M. E. Glasner, S. Yekta, C. B. Burge, and D. P. Bartel. 2003. Vertebrate microRNA genes. Science 299:1540.

Mello, C. C., and M. P. Czech. 2004. Micromanaging insulin secretion. Nat. Med. 10:1297-1298.

Mersey, B. D., P. Jin, and D. J. Danner. 2005. Human microRNA (miR29b) expression controls the amount of branched chain alpha-ketoacid dehydrogenase complex in a cell. Hum. Mol. Genet. 14:3371-3377.

Naeem, A., K. Zhong, S. J. Moisa, J. K. Drackley, K. M. Moyes, and J. J. Loor. 2012. Bioinformatics analysis of microRNA and putative target genes in bovine mammary tissue infected with Streptococcus uberis. J. Dairy Sci. 95:6397-6408.

Nan, X., D. Bu, X. Li, J. Wang, H. Wei, H. Hu, L. Zhou, and J. J. Loor. 2014. Ratio of lysine to methionine alters expression of genes involved in milk protein transcription and translation and mTOR phosphorylation in bovine mammary cells. Physiol. Genomics $46: 268-275$.

Oshlack, A., and M. J. Wakefield. 2009. Transcript length bias in RNA-seq data confounds systems biology. Biol. Direct 4:14.

Romao, J. M., W. Jin, M. He, T. McAllister, and L. L. Guan. 2012. Altered microRNA expression in bovine subcutaneous and visceral adipose tissues from cattle under different diet. PLoS ONE $7: \mathrm{e} 40605$

Skalsky, R. L., D. L. Corcoran, E. Gottwein, C. L. Frank, D. Kang, M. Hafner, J. D. Nusbaum, R. Feederle, H. J. Delecluse, M. A. Luftig, T. Tuschl, U. Ohler, and B. R. Cullen. 2012. The viral and cellular microRNA targetome in lymphoblastoid cell lines. PLoS Pathog. 8:e1002484.

Sun, J., M. Li, Z. Li, J. Xue, X. Lan, C. Zhang, C. Lei, and H. Chen. 2013. Identification and profiling of conserved and novel microRNAs from Chinese Qinchuan bovine longissimus thoracis. BMC Genomics 14:42.

Trompeter, H. I., H. Abbad, K. M. Iwaniuk, M. Hafner, N. Renwick, T. Tuschl, J. Schira, H. W. Muller, and P. Wernet. 2011. MicroRNAs MiR-17, MiR-20a, and MiR-106b act in concert to modulate E2F activity on cell cycle arrest during neuronal lineage differentiation of USSC. PLoS ONE 6:e16138.

Valencia-Sanchez, M. A., J. Liu, G. J. Hannon, and R. Parker. 2006. Control of translation and mRNA degradation by miRNAs and siRNAs. Genes Dev. 20:515-524.

Wang, M., S. Moisa, M. J. Khan, J. Wang, D. Bu, and J. J. Loor. 2012. MicroRNA expression patterns in the bovine mammary gland are affected by stage of lactation. J. Dairy Sci. 95:6529-6535.

Wang, X., L. Ye, Y. Zhou, M. Q. Liu, D. J. Zhou, and W. Z. Ho. 2011. Inhibition of anti-HIV microRNA expression: a mechanism for opioid-mediated enhancement of HIV infection of monocytes. Am. J. Pathol. 178:41-47.

Zhu, H., N. Shyh-Chang, A. V. Segre, G. Shinoda, S. P. Shah, W. S. Einhorn, A. Takeuchi, J. M. Engreitz, J. P. Hagan, M. G. Kharas, A. Urbach, J. E. Thornton, R. Triboulet, R. I. Gregory, D. Altshuler, and G. Q. Daley. 2011. The Lin28/let-7 axis regulates glucose metabolism. Cell 147:81-94. 